\title{
High speed millimeter-wave and visible light communication with off-the-shelf components
}

\author{
Iman Tavakkolnia ${ }^{1} *$, David Cheadle ${ }^{2}$, Rui Bian ${ }^{3}$, Tian Hong Loh $^{2}$, and Harald Haas ${ }^{1}$ \\ ${ }^{1}$ LiFi Research \& Development Centre, The University of Strathclyde, Glasgow, UK. \\ ${ }^{2}$ National Physical Laboratory (NPL), Teddington, UK. \\ ${ }^{3}$ PureLiFi Ltd, Edinburgh, UK. \\ *e-mail: i.tavakkolnia@strath.ac.uk
}

\begin{abstract}
The ever increasing demand for higher data rates and seamless wireless connectivity have forced both academic and industrial strategists of wireless communications to consider exploring higher frequency bands other than the heavily utilised sub-6 GHz radio spectrum. The millimetre, infrared, and visible light spectra of electromagnetic waves are three of the most favourable choices. In this paper, we examine two wireless communication testbed systems operating in the millimetrewave (mmWave) and visible spectra. Both systems are based on commercially available off-the-shelf components. Different aspects, e.g., modulation, link distance and partial link blockage, are studied. We experimentally show that both systems are capable of providing giga-bit-per-second data rates, and infer that a hybrid system can be the solution that mitigates drawbacks of each individual technology.
\end{abstract}

Index Terms-Orthogonal frequency division multiplexing (OFDM), Light Fidelity (LiFi), Millimeter-wave (mmWave), Visible light communication (VLC), 5G.

\section{INTRODUCTION}

There is no doubt that new communication technologies are required for future wireless networks to cope with the ever increasing data rate demands [1]. The compound annual growth rate (CAGR) of wireless traffic in the past years has remained at a constant rate of $60 \%$ [2]. This growth will not slow down in the foreseeable future due to the constant increase of generated data (e.g., high quality video stream and cloud-based gaming) and technologies such as Internetof-Things (IoT) and machine type communication (MTC). Therefore, electromagnetic spectra other than the sub- $6 \mathrm{GHz}$ frequency band, such as millimeter wave (mmWave), infrared and visible light, are being explored. The new technologies are not intended to replace the existing ones, but they will be deployed gradually in the most suitable scenarios to offload a significant portion of the data traffic. Moreover, some of the future technologies, such as vehicle-to-vehicle $(\mathrm{V} 2 \mathrm{~V})$ communication, can be designed based on these new spectra. The available and unregulated wide bandwidths in the

This work was supported by Royal Academy of Engineering and Newton Fund under project reference UK-CIAPP/369. Professor H. Haas gratefully acknowledges the support by the Engineering and Physical Research Council (EPSRC) under Grant EP/R007101/1. He also acknowledges the financial support by the Wolfson Foundation and the Royal Society. The work of D. Cheadle and T. H. Loh was in part supported by The 2017-2020 National Measurement System Programme of the UK government's Department for Business, Energy and Industrial Strategy (BEIS), under Science Theme Reference EMT20 of that Programme.
mmWave, infrared and visible light spectra have made the corresponding technologies extremely promising for data capacity enhancement. Consequently, research in these technologies has significantly increased in the last decade and industry has started to commercialize these technologies [3], [4].

The mmWave spectrum is considered for wireless communication due to the large available bandwidth and some specific characteristics such as the strong oxygen-induced atmospheric attenuation that enables small cells [5]. The $60 \mathrm{GHz}$ mmWave band is selected for standards IEEE802.11ad, known as WiGig, and IEEE 802.15.3c task group for the physical layer (PHY) of wireless personal area networks (WPAN). Moreover, different aspects of wireless communication in the mmWave bands, e.g., channel modelling, signal processing and system design, have been the subject of an immense amount of research, and efforts have been made to include it in $5 \mathrm{G}$ cellular networks and other applications such as autonomous vehicles [6]. Many physical phenomena are introduced due to the higher frequencies, which make the mmWave system different from the sub-6 $\mathrm{GHz}$ communication bands [7]. For instance, the free space loss is higher for mmWave due to its proportionality to carrier frequency; gas molecules, rain droplets, and presence of vegetation can attenuate the propagating waves in the mmWave spectrum; and the propagation mechanisms, such as penetration through obstacles, diffuse scattering and diffraction properties, become significantly important due to the short wavelength of mmWave signals. Therefore, a number of analytical and measurement-based channel models have been presented over the past years for various indoor and outdoor scenarios, and high speed communication links have been demonstrated [3], [8].

While $300 \mathrm{GHz}$ of bandwidth (including mmWave) is available in the entire radio frequency (RF) spectrum, visible light $(380-780 \mathrm{~nm})$ alone consists of about $400 \mathrm{THz}$ of bandwidth. Including infrared (IR), the light spectrum is 2600 times the size of the entire RF spectrum [9]. Therefore, the huge potential of the light spectrum becomes evident, noting in particular that the basic infrastructure, i.e., light emitting diodes (LEDs), are ubiquitous. Visible light communication (VLC) is considered for standards such as IEEE 802.15.7 and $802.11 \mathrm{bb}$ task groups. Light-fidelity (LiFi) was introduced in 2011 and was defined as a bidirectional, multi-user, and high-speed wireless communication network [10]. The nature of light waves imposes special considerations for the design 
of a VLC system. For instance, enhanced security is an advantage of light blockage by opaque objects, but at the same time, communication links are affected adversely. In the literature, channel models and transceiver structures have been extensively studied [11]-[13], high data rates are reported by using inexpensive off-the-shelf components [14].

The mmWave and visible light spectra are different in nature, but at the same time, they exhibit some similar characteristics as they both operate at higher frequencies compared to traditional RF systems [15]. Therefore, a hybrid ecosystem can ideally be developed for future wireless communication systems that includes both technologies. In this paper, as a first step, we have experimentally analysed and compared the two testbed systems operating with mmWave and VLC, both with relevant commercially available off-the-shelf components. Such systems can be used for point-to-point communication, such as backhaul connectivity. The experiments are performed in a controlled manner (i.e., in terms of power, bandwidth, modulation order) in a shielded indoor laboratory environment. However, different types of orthogonal frequency division multiplexing (OFDM) are utilised as the modulation technique due to the inherent frequency response difference of two systems. It is shown that even these simple off-theshelf systems can achieve giga-bit-per-second data rates. It has also been demonstrated that different materials may affect one system more than the other, and thus, a robust hybrid system is needed to be designed based on the inherent nature of the two technologies.

The rest of the paper is organized as follows. In Section II, a brief overview and channel models of the two systems are presented. The experimental set-up is described in Section III, and the performance evaluation results are explained in Section IV. Finally, Section V concludes the paper.

\section{SYSTEM OVERVIEW}

In this section, a brief overview of key aspects of communication in mmWave and visible light spectra are presented and references are introduced for more details.

\section{A. mmWave}

The traditional definition of mmWave covers the frequency range between $30 \mathrm{GHz}$ to $300 \mathrm{GHz}$. Nevertheless, the recent trends on exploring various potential frequency candidates for $5 \mathrm{G}$ communications have resulted in frequencies above 24 $\mathrm{GHz}$ being considered as part of mmWave bands [16]. In fact, the $26 \mathrm{GHz}$ band has recently been identified as the candidate frequency for UK 5G deployment [17], which is also chosen for this paper. The existence of a line-of-sight (LoS) link is usually required for an acceptable communication performance due to high directionallity of the propagation. At the same time, mmWaves may be significantly attenuated by the surrounding objects and users, and therefore, establishing a non-LoS (NLoS) communication becomes essential. Many measurements in different indoor, outdoor, LoS, and NLoS scenarios as well as simulation studies (e.g., ray tracing) have been conducted to provide a statistical channel model [3], [6],
[18]. A common statistical model for the average path loss at a distance $d$ for mmWave systems is presented as

$$
L[\mathrm{~dB}]=\alpha+10 \beta \log _{10}(d)+\gamma,
$$

where $\alpha$ and $\beta$ are the linear model parameters that can be estimated from real-world measurements. The effect of shadowing is included by the log-normal term $\gamma \sim \mathcal{N}\left(0, \sigma_{s}^{2}\right)$. For instance, in [19], the parameters in (1) were estimated based on least square fitting to the path loss measurements performed in an urban area of New York City. Note that (1) is a simple model, but its parameters can be modified accordingly to include effects such as small scale fading and antenna gains [6]. The choice of antenna, omni-directional or directional antennas, also affects the channel model and signal processing techniques. Moreover, due to the short wavelength of mmWaves, arrays of antennas can be designed with reasonable overall antenna size. Such antenna arrays are ideal for beamforming and multiple-input-multiple-output (MIMO) communication [20]. OFDM is already used for many standards, e.g., 5G, WPAN, and WiGig, because of its low complexity and robustness in frequency selective fading channel scenarios [21]. Therefore, we also consider OFDM as the modulation technique in this paper.

\section{B. $V L C$}

Due to the physics of LEDs, unlike lasers that are used for optical fibre and free space optical (FSO) communication, the phase of an optical wave cannot be used for data encoding. Therefore, VLC is based on intensity modulation (IM) and direct detection (DD) of optical signals. While this lowers the implementation complexity of VLC systems, conventional wireless communication techniques should be modified to be used in VLC. Moreover, the available modulation bandwidth of devices should be utilised efficiently to enable high data rates. Many modulation techniques have been proposed for VLC that satisfy the positive and real signal constraints imposed by the IM/DD. Direct current (DC) biased optical OFDM (DCO-OFDM) is a high performance low complexity technique that has enabled high data rate VLC with off-theshelf LEDs [14].

In order to generate a real time domain signal, the modulated data vector should follow a Hermitian symmetry. A constant DC value is then added to the signal to make it positive. Since an OFDM signal with a sufficiently large number of subcarriers follows a Guassian distribution, a few samples may still have a negative value that are clipped to zero. Also, the output optical power of an LED is limited, which leads to an upper clipping. As a result, the channel model including the LED nonlinearity and clipping effect is expressed as

$$
y(t)=h(t) * g(x(t))+n(t),
$$

where $x(t)$ and $y(t)$ are, respectively, the input and output signals. In (2), $h(t)$ is the optical channel response, $g(\cdot)$ is the nonlinear distortion, and $n(t)$ is the additive white Gaussian noise (AWGN) at the receiver with the double-sided power 
spectral density (PSD) of $N_{0}$. Applying the Bussgang theorem, the effect of nonlinear distortion $g(\cdot)$ can be expressed as an attenuation, by a constant factor $a$, accompanied by an uncorrelated distortion. This is given by

$$
g(x(t))=a x(t)+w(t),
$$

where $w(t)$ is a random process. In the frequency domain, by applying the central limit theorem, the noise can be modelled as a Gaussian process as $\mathcal{N}\left(0, \sigma_{w}^{2}\right)$. Therefore, the signal-tonoise ratio (SNR) at the $k$ th subcarrier with the overall channel gain $H_{k}$ is given as

$$
\rho_{k}=\frac{a^{2} H_{k}^{2} E_{k}}{N_{0}+H_{k}^{2} \sigma_{w}^{2}},
$$

where $E_{k}$ is the energy at subcarrier $k$. The channel gain $H_{k}$ depends on the optical and electrical properties of the system. This is usually dominated by the lowpass frequency response of electro-optic devices, i.e., the LED and photodetector, as well as the optical link itself. Detailed formulation of LOS and NLOS optical channel are given in many references such as [13].

Once we have the SNR, the channel capacity at the $k$ th subcarrier can be estimated as $C_{k}=\log \left(1+\rho_{k}\right)$. This exact capacity can only be achieved by complex modulation and coding techniques. Here, we consider quadrature amplitude modulation (QAM) accompanied by a suitable coding scheme, and use adaptive bit loading to utilize the system close to its information capacity. The adaptive bit loading algorithm is based on choosing the maximum number of bits that can be modulated at each subcarrier with a BER lower than the target [22]. For this purpose, a target bit error ratio (BER) is chosen that can be reduced significantly (i.e., effectively error free communication) by applying a specific forward error correction (FEC) scheme. In this paper, we use the target BER of $4.6 \times 10^{-3}$ that guarantees almost error free communication when a staircase coding scheme with $6.25 \%$ overhead is incorporated [23]. The BER at the $k$ th subcarrier with a QAM of order $M_{k}$ is given by [24]

$$
\begin{aligned}
& \operatorname{BER}\left(M_{k}, \rho_{k}\right) \simeq \frac{4\left(1-\frac{1}{\sqrt{M_{k}}}\right)}{\log _{2} M_{k}} \\
& \times \sum_{l=1}^{\min \left(2, \sqrt{M_{k}}\right)} \mathrm{Q}\left((2 l-1) \sqrt{\frac{3 \rho_{k}}{2\left(M_{k}-1\right)}}\right)
\end{aligned}
$$

where $\mathrm{Q}($.$) is the Gaussian Q-function. Based on the SNR$ value at each subcarrier, $\rho_{k}$, the maximum $M_{k}$ for all subcarriers are found so that $\operatorname{BER}\left(M_{k}, \rho_{k}\right) \leq 4.6 \times 10^{-3}$. Finally, the overall data rate is given by

$$
R=\frac{\sum_{k=1}^{\frac{N_{\mathrm{FFT}}}{2}} \log _{2} M_{k}}{\left(N_{\mathrm{FFT}}+N_{\mathrm{CP}}\right) / 2 B}
$$

where $B$ is the single-sided modulation bandwidth of the system, $N_{\mathrm{FFT}}$ is the number of subcarriers, and $N_{\mathrm{CP}}$ is the cyclic prefix size. Note that, in the experiment, the SNR at each subcarrier is estimated using a pilot OFDM signal and

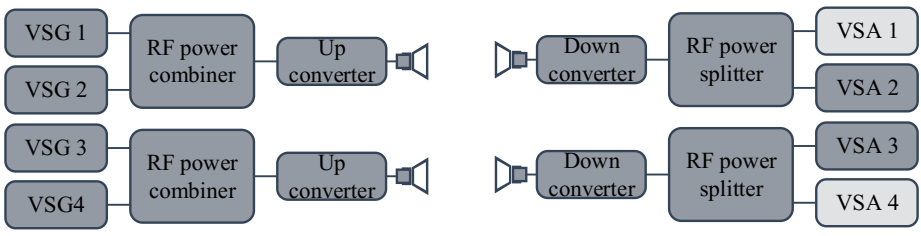

Fig. 1: mmWave system block diagram.

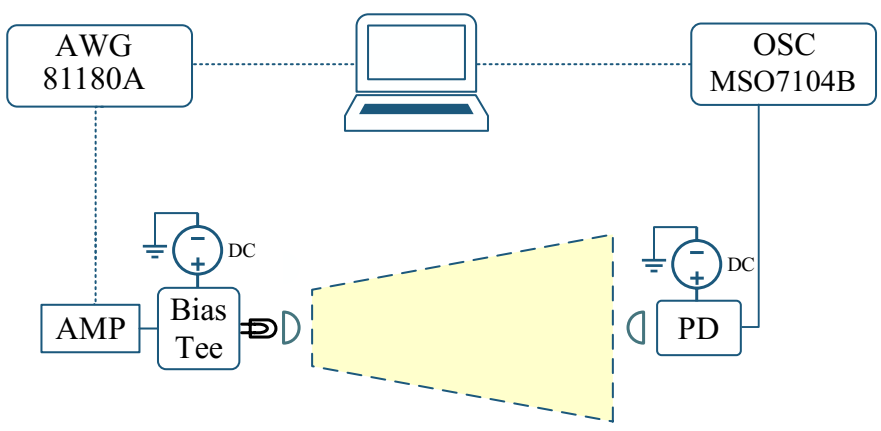

Fig. 2: VLC System block diagram.

a mean estimator. This takes into account every optical and electrical phenomena that may further affect the system, e.g., the imprecise optical alignment, the receiver responsivity, the amplifier nonlinearity, or the lowpass response of the oscilloscope. Then, several random OFDM signals are transmitted after applying the adaptive bit loading, and the actual BER is measured and confirmed to be below the target BER.

\section{EXPERIMENTAL SET-UP}

The block diagram of the mmWave system is depicted in Fig. 1 which is built around four vector signal transceivers (NI PXIe-5644R), each consisting of a vector signal generator (VSG) and analyser (VSA). The transceivers operate at an intermediate frequency (IF) which is converted to or from the RF frequency used for the transmission by mixing them with a local oscillator (LO) frequency. RF power combiners and splitters are used so that the same up and down convertors are incorporated for the two transceivers. This set-up provides carrier aggregation and allows the system to operate with a maximum of $480 \mathrm{MHz}$ of instantaneous bandwidth. Each transceiver typically operates with an instantaneous bandwidth of $80 \mathrm{MHz}$ for a total of $320 \mathrm{MHz}$ of occupied bandwidth and an RF operating frequency of $25.68 \mathrm{GHz}$ to $26 \mathrm{GHz}$. Two VSG and VSA are connected to each RF chain using power splitters/combiners. Four standard gain horn antennas (Flann 22240-20) with a typical gain of $20 \mathrm{~dB}$ were used in the system. The system transmits a random bit sequence which is modulated with OFDM and the symbols are mapped using up to 256-QAM. The sub carrier spacing is $120 \mathrm{kHz}$ and a maximum of 2640 subcarriers were used. The control software for the system is written in LabVIEW.

The block diagram of the VLC system is depicted in Fig. 2. We use a single commercial white light emitting diode (LED), VLMW1300, as the transmitter with $7.5 \mathrm{~mW}$ optical output power. The $3 \mathrm{~dB}$ bandwidth of this LED is $47 \mathrm{MHz}$. We use aspheric optical lenses (ACL4532) at both the transmitter 


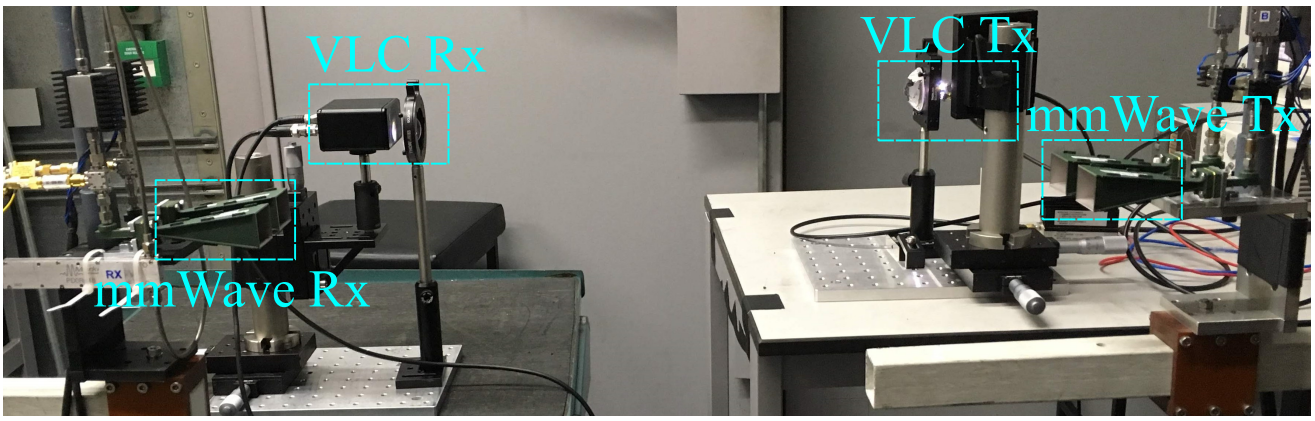

Fig. 3: System picture.

and receiver sides to guide the light through the link and use a single PIN photodiode (New Focus 1601 AC) as the receiver device. Random OFDM signals, with $N_{\mathrm{FFT}}=1024$ subcarriers, are generated in MATLAB software and sent to the arbitrary waveform generator (AWG, Keysight 81180A) for digital to analog conversion. In order to minimize the effect of LED nonlinearity, the OFDM signal is clipped at upper and lower clipping values of $3.2 \sigma_{x}$ and $-3.2 \sigma_{x}$, respectively, where $\sigma_{x}$ is the standard deviation of the OFDM waveform. This clipping range guarantees that the probability of signal clipping remains as small as $0.1 \%$ considering the Gaussian distribution of the OFDM signal. Different values of peak-to-peak voltages for the output of AWG are tested, and $600 \mathrm{mV}$ is chosen which leads to the maximum data rate. The signal is amplified using a high bandwidth high gain amplifier (Amp: Mini-Circuits ZHL-1A-S+) and fed into BiasTees (MiniCircuits ZFBT-4R2GW). The Bias-Tee is used to combine the OFDM signal and the DC bias provided by a DC power supply. The overall gain from AWG to the LED is about $13 \mathrm{~dB}$ including losses in components such as the bias-Tee. The LED is biased at a constant current of $100 \mathrm{~mA}$. At the receiver side, the output signal is captured by an oscilloscope (OSC, Keysight MSO7104B) and sent to a computer to be processed using MATLAB software.

The two systems explained above are put beside each other in a RF shielded lab, as shown in Fig. 3. The link distance for both systems is $1.1 \mathrm{~m}$ for all measurements except the one in which the effect of the link distance is studied. In both systems, random data is generated by the computer software and the system performance metrics are measured accordingly. The modulation bandwidth of the VLC system is limited by the modulation bandwidth of the white LED at a maximum of $660 \mathrm{MHz}$, while the bandwidth of the mmWave system is limited by the sampling rate of the FPGA used in the system at a maximum of $480 \mathrm{MHz}$ (120 MHz per FPGA). In the next section, different scenarios are defined and performances of the two systems are evaluated.

\section{REsults}

The normalised frequency responses of the two systems are first estimated using pilot sequences and are shown in Fig. 4. The frequency response of the mmWave system was measured using a vector network analyser (VNA) and numerically fitted using MATLAB. The frequency responses

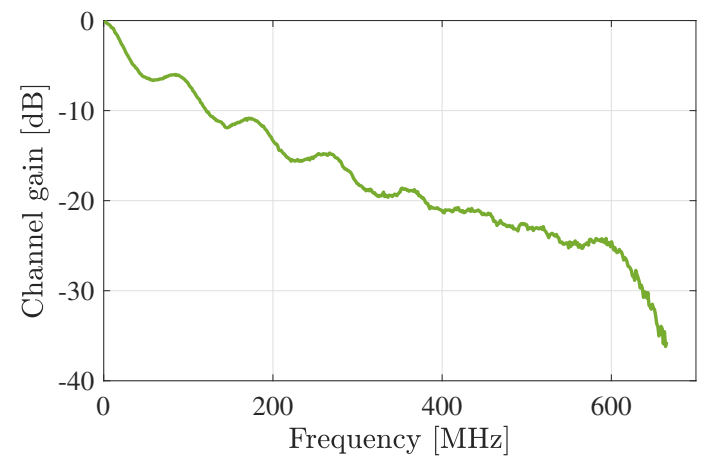

(a)

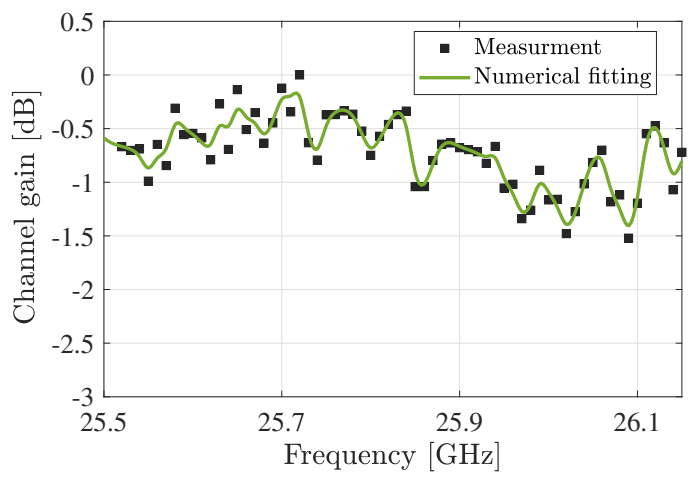

Fig. 4: Normalised frequency responses of (a) VLC (measurement) and (b) mmWave systems (Measurement and numerical fitting).

are shown in baseband and passband, respectively for VLC and mmWave systems, because of the difference in the used communication techniques. It can be seen that the channel for VLC is frequency-selective and lowpass, while the channel for mmWave is almost flat. This implies that adaptive bit loading is essential for VLC while a constant modulation order can be used for mmWave. We first evaluate this by changing the modulation orders for the two systems and also by incorporating adaptive bit loading for the VLC system. The results, data rate and BER, are shown in Fig. 5. The modulation bandwidth and input power are fixed at $320 \mathrm{MHz}$ and $10 \mathrm{dBm}$, respectively. Therefore, the data rate increases linearly when the modulation order changes. Adaptive bit loading is used for VLC with two different target BER values. It is observed that the mmWave 


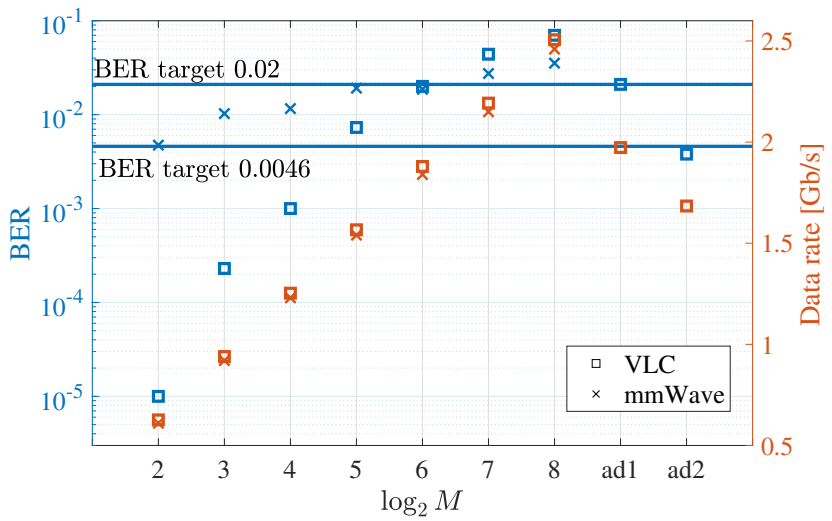

Fig. 5: Data rate and BER results for different modulation orders $M$. "ad1" and "ad2" refer to adaptive modulation with target BERs of 0.02 and 0.0046 , respectively.

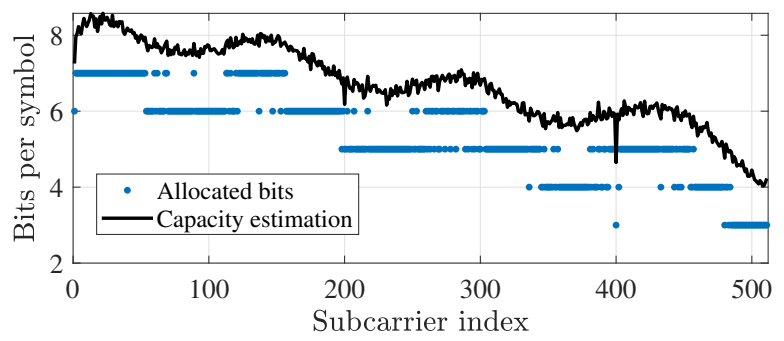

Fig. 6: Adaptive bit loading and capacity estimation results for the VLC system at a target BER of 0.0046 .

system requires advanced error correction coding schemes with potentially large overheads (e.g., about $33 \%$ at a target BER of 0.02 [23]). However, the VLC system demonstrates lower BER with a fixed modulation order and high data rate with adaptive modulation. The bit loading results are presented in Fig. 6 along with the capacity limit calculated using the estimated SNR (i.e., Shannon capacity) which confirms that this scheme performs close to the capacity limits of the system and is therefore nearly optimal.

In another set of measurements, the effect of link distance is studied for the two systems at a modulation bandwidth of $320 \mathrm{MHz}$. The results are shown in Fig. 7. The performance of the fixed modulation order 64-QAM is roughly the same for VLC and mmWave at any distance. However, VLC performs better at a lower modulation order 16-QAM which allows shorter coding overhead for error correction. The adaptive bit loading technique is also used and the result shows that a constant BER performance is achievable while the data rate decreases gradually. Fig. 7 shows that the BER does not follow a persistent trend for 16-QAM and adaptive bit loading, which is due to the slightly different alignment conditions for VLC, and the effect of surrounding environment for mmWave. It is important to emphasise that over $1 \mathrm{~Gb} / \mathrm{s}$ is easily achievable at any link distance . Due to limited space in the lab, longer distances were not experimented, but based on Fig. 7 and results from other works, a similar performance is also expected at longer distances.

In order to show the behaviour of two systems in case there

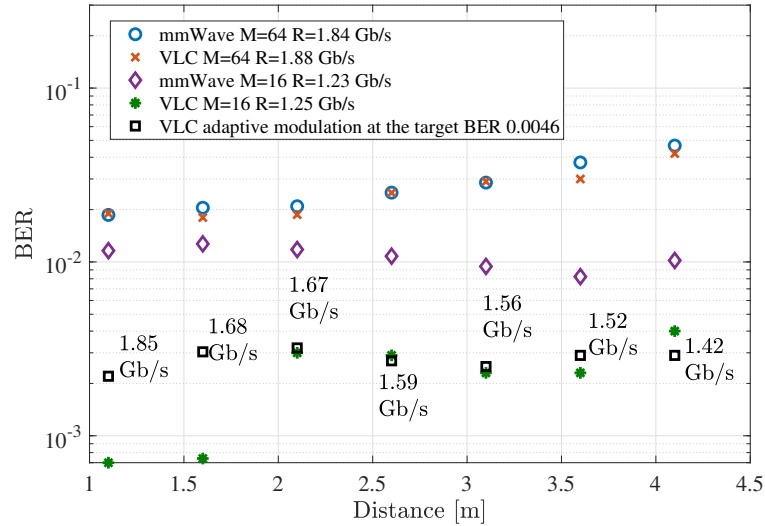

Fig. 7: Data rate and BER results for different link distances. is blockage, more experiments are performed. As expected, an opaque object completely blocks the LoS light beam, and the mmWave beams are blocked by metal objects depending on the wave parameters and potential holes in the object [25]. In this paper, we test a semi-opaque object, i.e., bubble wrap, and a copper mesh surface. Both links are blocked by the two objects, and results are demonstrated in Fig. 8. Pictures of receivers in the two scenarios are also included. It is observed that the VLC system is only slightly affected by the copper mesh while the BER increases an order of magnitude for the mmWave system. This happens because a considerable portion of the light passes through the mesh, while the mesh holes are not large enough for the mmWave signal to pass through. However, the BER and data rate results are significantly degraded for the VLC system in the case of the semi-opaque object blockage, whereas the mmWave system performs without any impact. Only adaptive bit loading can be used for VLC in this case with more than $1 \mathrm{~Gb} / \mathrm{s}$ loss of data rate. Note that these materials are chosen only for demonstration purposes in these scenarios, where the link for one of the two systems is almost completely blocked. In real applications, other materials and types of blockage may be seen that affect each system in a different manner. However, this experiment shows that due to the natural specifications of the two wavelengths, a hybrid system would form a robust solution. In other words, an intelligent system can be developed that uses both technologies at the same time or switches between the two in certain scenarios when one does not perform to the required levels. This is the subject of a future work.

\section{CONCLUSion}

Two typical mmWave and visible light communication systems with only off-the-shelf components were experimentally studied in this paper. Communication system parameters were controlled, and the two systems were compared in terms of frequency response, data rate, BER, and vulnerability to partial blockage. It was demonstrated that both systems can easily provide giga-bit-per-second data rate, while responding to link blockage in different manners. Therefore, we conclude that a hybrid mmWave/VLC system is a robust solution for 


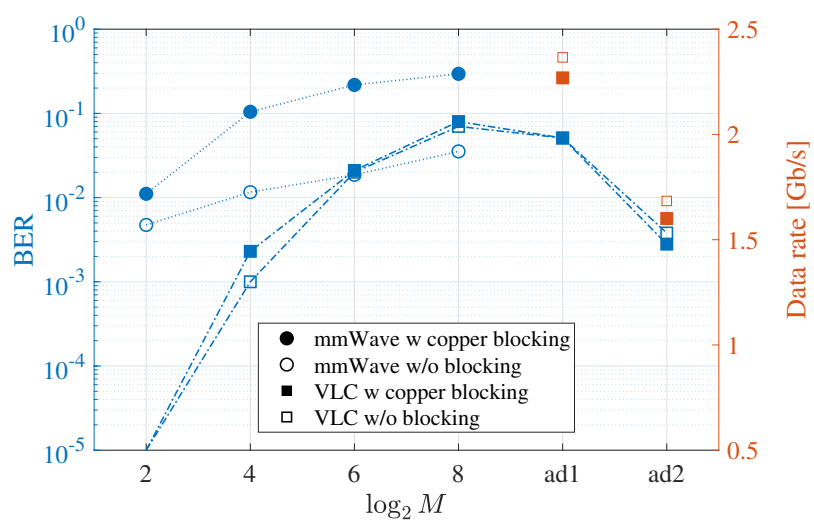

(a)

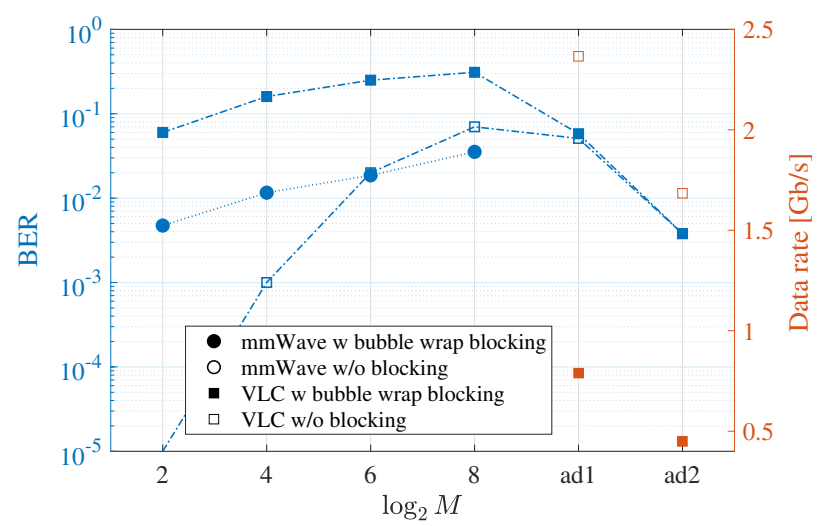

(b)

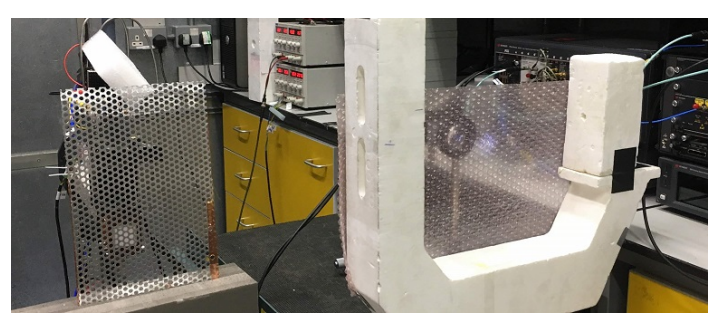

(c)

Fig. 8: Performance results for different blocking materials at $320 \mathrm{MHz}$ modulation bandwidth (a) copper sheet (b) bubble wrap. "ad1" and "ad2" refer to adaptive modulation with target BERs of 0.02 and 0.0046 , respectively. Data rates for modulation orders $M=4,16,64,256$ are respectively $0.6,1.2,1.8,2.5 \mathrm{~Gb} / \mathrm{s}$. (c) Pictures of receivers with blockage.

deployment for different applications such as high speed backhaul links.

\section{REFERENCES}

[1] H. Haas, "LiFi is a paradigm-shifting $5 \mathrm{G}$ technology," Reviews in Physics, vol. 3, pp. 26-31, Nov. 2018.

[2] P. J. Winzer and D. T. Neilson, "From scaling disparities to integrated parallelism: A decathlon for a decade," J. Lightw. Technol., vol. 35, no. 5, pp. 1099-1115, 2017.

[3] I. A. Hemadeh, K. Satyanarayana, M. El-Hajjar, and L. Hanzo, "Millimeter-wave communications: Physical channel models, design considerations, antenna constructions, and link-budget," IEEE Commun. Surveys Tut., vol. 20, no. 2, pp. 870-913, Dec. 2017.

[4] I. Demirkol, D. Camps-Mur, J. Paradells, M. Combalia, W. Popoola, and H. Haas, "Powering the internet of things through light communication," IEEE Commun. Mag., vol. 57, no. 6, pp. 107-113, May 2019.
[5] F. Giannetti, M. Luise, and R. Reggiannini, "Mobile and personal communications in the $60 \mathrm{GHz}$ band: A survey," Wireless Personal Communications, vol. 10, no. 2, pp. 207-243, Jul. 1999.

[6] R. W. Heath, N. Gonzalez-Prelcic, S. Rangan, W. Roh, and A. M. Sayeed, "An overview of signal processing techniques for millimeter wave MIMO systems," IEEE J. Sel. Topics Signal Process., vol. 10, no. 3, pp. 436-453, Feb. 2016.

[7] Z. Pi and F. Khan, "An introduction to millimeter-wave mobile broadband systems," IEEE Commun. Mag., vol. 49, no. 6, pp. 101-107, Jun. 2011.

[8] C. Dehos, J. L. González, A. De Domenico, D. Ktenas, and L. Dussopt, "Millimeter-wave access and backhauling: The solution to the exponential data traffic increase in 5G mobile communications systems?" IEEE Commun. Mag., vol. 52, no. 9, pp. 88-95, Sep. 2014.

[9] H. Haas, J. Elmirghani, and I. White, "Optical wireless communication," Phil. Trans. R. Soc. A, vol. 378, no. 20200051, pp. 1-11, Mar 2020.

[10] H. Haas, L. Yin, Y. Wang, and C. Chen, "What is LiFi?" J. Lightw. Technol., vol. 34, no. 6, pp. 1533-1544, Mar. 2016.

[11] F. Miramirkhani and M. Uysal, "Channel modelling for indoor visible light communications," Phil. Trans. R. Soc. A, vol. 378, no. 2169, p. 20190187, Apr. 2020.

[12] M. A. Arfaoui, M. D. Soltani, I. Tavakkolnia, A. Ghrayeb, C. Assi, M. Safari, and H. Haas, "Measurements-based channel models for indoor lifi systems," arXiv preprint arXiv:2001.09596, 2020.

[13] M. D. Soltani, M. A. Arfaoui, I. Tavakkolnia, A. Ghrayeb, M. Safari, C. M. Assi, M. O. Hasna, and H. Haas, "Bidirectional optical spatial modulation for mobile users: Toward a practical design for LiFi systems," IEEE J Sel. Areas Commun., vol. 37, no. 9, pp. 2069-2086, Aug. 2019.

[14] R. Bian, I. Tavakkolnia, and H. Haas, " $15.73 \mathrm{~Gb} / \mathrm{s}$ visible light communication with off-the-shelf LEDs," J. Lightw. Technol., vol. 37, no. 10, pp. 2418-2424, Mar. 2019.

[15] L. Feng, H. Yang, R. Q. Hu, and J. Wang, "MmWave and VLCbased indoor channel models in 5G wireless networks," IEEE Wireless Commun., vol. 25, no. 5, pp. 70-77, Aug. 2018.

[16] Qualcomm: Breaking the wireless barriers to mobilize $5 G \mathrm{NR}$ mmWave, Qualcomm, United States, May 2019. [Online]. Available: https://www.qualcomm.com/media/documents/files/5g-nr-mmwavedeployment-strategy-presentation.pdf.

[17] Ofcom: Enabling $5 G$ in th $U K$, Ofcom, London, United Kingdom, Mar. 2018. [Online]. Available:https://www.ofcom.org.uk/_data/assets/pdf_file /0022/111883/enabling-5g-uk.pdf.

[18] G. R. Maccartney, T. S. Rappaport, S. Sun, and S. Deng, "Indoor office wideband millimeter-wave propagation measurements and channel models at 28 and $73 \mathrm{GHz}$ for ultra-dense 5G wireless networks," IEEE Access, vol. 3, pp. 2388-2424, Oct. 2015.

[19] M. R. Akdeniz, Y. Liu, M. K. Samimi, S. Sun, S. Rangan, T. S. Rappaport, and E. Erkip, "Millimeter wave channel modeling and cellular capacity evaluation," IEEE J. Sel. Areas Commun., vol. 32, no. 6, pp. 1164-1179, Jun. 2014.

[20] S. A. Busari, K. M. S. Huq, S. Mumtaz, L. Dai, and J. Rodriguez, "Millimeter-wave massive MIMO communication for future wireless systems: A survey," IEEE Commun. Surveys Tut., vol. 20, no. 2, pp. 836-869, Dec. 2017.

[21] C. Sacchi, T. F. Rahman, I. A. Hemadeh, and M. El-Hajjar, "Millimeterwave transmission for small-cell backhaul in dense urban environment: A solution based on MIMO-OFDM and space-time shift keying (STSK)," IEEE Access, vol. 5, pp. 4000-4017, Mar. 2017.

[22] O. Narmanlioglu, R. C. Kizilirmak, T. Baykas, and M. Uysal, "Link adaptation for MIMO OFDM visible light communication systems," IEEE Access, vol. 5, pp. 26006-26014, Nov. 2017.

[23] L. M. Zhang and F. R. Kschischang, "Staircase codes with $6 \%$ to $33 \%$ overhead," J. Lightw. Technol., vol. 32, no. 10, pp. 1999-2002, May 2014.

[24] D. Tsonev, S. Videv, and H. Haas, "Unlocking spectral efficiency in intensity modulation and direct detection systems," IEEE J. Sel. Areas Commun., vol. 33, no. 9, pp. 1758-1770, Sep. 2015.

[25] K. F. Casey, "Electromagnetic shielding behavior of wire-mesh screens," IEEE Trans. Electromagn. Compat., vol. 30, no. 3, pp. 298-306, Aug. 1988. 